\title{
Comparision of snowball sampling and sequential sampling technique
}

\begin{abstract}
The article provides the description and comparison of two non-random samplings which are snowball or chain referral sampling and sequential sampling. Snowball sampling has been widely used in qualitative sociological research, especially in the study of deviant behavior and is used in the place where the population is hard to reach. It also described different form of sampling method. While in sequential sampling, sampling was taken at a given time interval and modification can be made by correcting the research and sampling method to centralize the analysis and make a satisfied decision.
\end{abstract}

Volume I Issue 3 - 2016

\author{
Ilker Etikan, Rukayya Alkassim, Sulaiman \\ Abubakar \\ Department of Biostatistics, Near East University, Cyprus
}

Correspondence: Ilker Etikan, Near East University, NicosiaTRNC, Cyprus, Email ietikan@gmail.com

Received: November 18, 2015 | Published: January 02, 2016

\section{Introduction}

Representing against population with a subset of it is termed as sampling. Sampling can either be statistical or non-statistical. In statistical sampling (probability sampling technique) calculating the probability of getting any particular sample is possible. It is scientific and every element stands an equal chance of being selected. In statistical sampling, workforce, time and money highly limits most researchers from getting true random sample that will represent the entire population. Thus, a non-probability sampling techniques are needed. Non-probability sampling techniques help researchers to subjectively select unit that represents the population under study. Unlike probability sampling, non-probability sampling does not involve random selection rather samples are selected based on accessibility. In applied social researches, non probabilistic methods are used especially when random sampling is not theoretically, practically and feasibly sensible. This paper will review two exemplary non-probability sampling techniques, namely; snowball sampling and sequential sampling met

\section{Snowball sampling}

Snowball sampling or Chain-referral-sampling of a hidden population begins with a convenience sample of initial subject, because if a random sample could be drawn, the population would not restrict as hidden. This initial subject serve as "seeds," through which wave 1 subject is recruited; wave 1 subject in turn recruit wave 2 subjects; and the sample consequently expands wave by wave like a snowball growing in size as it rolls down a hill. ${ }^{1}$

This sampling method generates biased samples because respondents who have great number of social connections are able to provide investigators with a higher proportion of other respondents who have characteristics similar to that initial respondent. ${ }^{2}$ The impossibility of making unbiased estimate from snowball samples was believed, but snowball sampling variation is called respondentdriven sampling. It allows the researcher to make asymptotically unbiased estimates from snowball samples under some conditions. ${ }^{3}$ Hence Snowball sampling and respondent-driven sampling allow participants to make estimates about the social network connecting the hidden population.

One of the most well-known forms of non-probability sampling is the snowball sampling method, which is particularly suitable when the population of interest is hard to reach and compiling a list of the population poses difficulties for the researcher. Common examples of the use of snowball sampling involve sociological studies into hidden populations that may be involved in sensitive issues or illegal activities, such as drug use and prostitution. ${ }^{4}$

As with random sampling, the snowballing method is not as uncontrolled as its name implied. The researcher is deeply involved in developing and managing the origination and progress of the sample, and seeks to ensure at all times that the chain of referrals remains within limitations that are relevant to the study. One of the dangers with snowball sampling is that respondents often suggest others who share similar characteristics, or the same outlook, and it is also compulsory on the researcher to ensure that the initial set of respondents is sufficiently varied so that the sample is not skewed excessively in any one particular direction.

\section{Types of snowball sampling}

Linear snowball sampling is a snowball sampling in which the researcher recruits a single participant, while the second nominee recruits the third participant. The chain continues to refer linearly up to the end of the sampling. The second type is Exponential NonDiscriminative Snowball Sampling where every recruited participant in the research work recruits another participant while in the exponential discriminative snowball sampling not every recruited participant is going to recruit another participant; the chain is discriminating. ${ }^{5}$ One of the most important uses of this technique is the possibility for the researchers to comprise people in the survey that they would not have known locating members of a specific population.

Robert ${ }^{6}$ used snowball sampling to study homeless persons living in Santa Barbara, California: I began this process by attending a meeting of homeless people I had heard about through my housing advocate contacts. . . . One homeless woman ... invited me to . . . where she promised to introduce me around. Thus a process of snowballing began. I gained entree to a group through people I knew, came to know others, and through them gained entree to new circles. ${ }^{6}$

As the subject is used to locate the hidden population, the researcher doesn't have to invest money and time for the sampling process. Snowball sampling method doesn't require a multifarious planning and the staff used is considerably smaller in comparison to other sampling methods.

One problem with this technique is that the initial contacts may form the entire sample and exclude access to some members of the 
population of interest. However, the main challenge of sampling a hidden population is those are mark out usually, and practicing illegal behaviors make the participants difficult to access them and reluctant to participate in the research.

\section{Sequential sampling}

This is a complex sample design where the fundamental sample size is not fixed in advance but is determined according to mathematical decisions on the basis of information yielded as survey progresses. This design is usually accepted under acceptance sampling plan in the field of statistical quality control. In practice, in the probability sampling the methods of sampling described above may well be used in the same study in which case it can be called mixed sampling. It may be pointed out here that normally one should resort to random sampling so that bias can be eliminated and sampling error can be estimated. But purposive sampling is considered desirable when the universe happens to be small and a known characteristic of it is to be studied intensively. Also, there are conditions under which sample designs other than random sampling may be considered better for reasons like convenience and low costs. The sample design to be used must be decided by the researcher taking into thought, the nature of the inquiry and other related factors. ${ }^{7}$

Sequential sampling is a non-probability sampling technique in which the researcher picks a single or a group of population in a given time interval, performs his study, analyzes the results then picks another group of population if needed and so on. This sampling technique gives the researcher boundless chances of fine tuning his research methods and gaining a vigorous awareness into the study that he is currently following. ${ }^{5}$

In sequential sampling, if the probability of rejecting $\mathrm{H}_{0}$ (the null hypothesis) when $\mathrm{H}_{0}$ is true (Type I Error) or ( $\left.\boldsymbol{\alpha}\right)$ and the probability that $\mathrm{H}_{0}$ will be accepted when $\mathrm{H}_{1}$ (the alternative hypothesis) is true (Type II Error) or ( $\boldsymbol{\beta})$ are chosen in advance and the sample size is allowed to differ. The sample is drawn one unit at a given time, and at each draw a decision, is made. The null hypothesis may be accepted, rejected, or no decision reached. If no decision is reached, the sampling is continued. If either of the other events occurs, sampling is terminated and a decision is reached at the $\boldsymbol{\alpha}$ and $\boldsymbol{\beta}$ confidence levels. ${ }^{8}$ Before a sequential sampling scheme can be applied to estimate the density of a given population in biological work, Binomial distribution and the Normal distribution are the two distributions commonly come across. ${ }^{8}$

One of the major advantages of sequential sampling is Limitless option when it comes to sample size selection and sampling schedule, Not expensive and no time consuming, Not workforce extensive and Minor changes and adjustments can be done during the initial part of the study to correct and hone the research method.
However, the sampling method cannot use to represent the entire population, only when large sample size was chosen and it cannot use to create conclusion or make decision to the entire population.

\section{Conclusion}

Both snowball and sequential sampling are non-random sampling because not every element in the population has equal chance of being selected as the sample. Snowball sampling is used where potential participants are hard to locate. The method is well suited for a number of research purposes and is particularly applicable when the focus of study is on a subtle issue, possibly concerning a relatively secretive matter, and thus requires the knowledge of insiders to locate people for the study and it is impossible to determine the sampling error or make inference about the population on the samples obtained. But in sequential sampling, the sample was taken at a given time interval, adjustment can be made by correcting the research and sampling method to centralize the analysis and make a satisfied decision, hence sequential sampling techniques is more preferred than snowball sampling.

\section{Acknowledgement}

None.

\section{Conflict of interest}

None.

\section{References}

1. Heckathorn DD. Snowball Versus Respondent-Driven Sampling. Sociol Methodol. 2015;41(1):352-366.

2. Erickson BH. Some Problem of from Chain Data. Annal of Mathematical Statistics. 1979;270-302.

3. Jonhston LG, Keith S. Sampling Hard To reach Population with Resspondent-Driven sampling. Methodological Innovations Online. 2010;5(2):38-48.

4. Oisín T. Process tracing and Elite Interviewing: A case for non-probability sampling. Political Science and Politics. 2007;40(4):481-496.

5. https://explorable.com/sequential-sampling. 2010.

6. Robert R. Writing Meta-Analytic Review. The American Psychological Association. 1995;118(2):183-192.

7. Jawale KV. Methods of Sampling Design in the Legal Research Advantages and Disadvantages. Online International Interdisciplinary Research Journal. 2012;183-190.

8. Ives WG. Sequential Sampling of insect Populations. 1950;287-291. 